\title{
Picosecond Time-Resolved Fourier Transform Raman Spectroscopy of 9,10-Diphenylanthracene in the Excited Singlet State
}

\author{
GOURI S. JAS, CHAOZHI WAN, and CAREY K. JOHNSON* \\ Department of Chemistry, University of Kansas, Lawrence, Kansas 66045
}

\begin{abstract}
Time-resolved Fourier transform Raman spectroscopy of the highly fluorescent chromophore 9,10-diphenylanthracene (DPA) in cyclohexane and ethanol is described. Raman spectra of the first excited singlet state of DPA were obtained with 100 -ps resolution at several time delays between pump pulses at $355 \mathrm{~nm}$ and probe pulses at $1064 \mathrm{~nm}$. The nearinfrared excited-state Raman scattering is enhanced by resonance with an excited-state transition of DPA. The excited-state Raman bands decay in about 5-6 ns. Evidence for interaction of the solvent with the DPA excited state is observed in the cyclohexane $\mathrm{C}-\mathrm{H}$ stretching bands.
\end{abstract}

Index Headings: Time-resolved Raman; FT-Raman; Step-scan FTRaman; Excited states.

\section{INTRODUCTION}

For many years, spectroscopists have sought to apply the methods of vibrational spectroscopy to excited states, photochemical intermediates, and transient ground-state species. Consequently, time-resolved resonance Raman spectroscopy ${ }^{1-6}$ has been an area of rapid development in recent years. However, like conventional resonance Raman spectroscopy, time-resolved resonance Raman methods have sometimes been hampered by fluorescent background. Although it is possible in some cases to deal with this problem by careful subtraction of backgrounds recorded with pump and probe beams alone, ${ }^{7}$ this method corrects only for the average background levels, and does not remove the noise component. Hence, in most cases, time-resolved Raman methods have been applied either to samples with low fluorescence quantum yields or to samples where excited-state Raman scattering generated by the probe beam is spectrally removed from the fluorescence induced by the pump beam. This approach can potentially be achieved in samples with visible or UV fluorescence by Raman excitation and detection in the near-infrared region. In recent years, the combination of Fourier transform (FT) detection and analysis with nearIR excitation of Raman scattering has proven to be a particularly powerful technique for steady-state Raman spectroscopy.$^{8-12}$ The development of near-IR FT-Raman instrumentation also furnishes the opportunity to expand the spectral region available for time-resolved Raman experiments into the near-infrared.

The use of FT methods with an externally modulated signal requires simultaneous synchronization of the interferometer mirror position and the external modulation. This constraint also applies to time-resolved signals that are generated by short laser pulses. An elegant and

Received 6 May 1994; accepted 6 February 1995.

* Author to whom correspondence should be sent. flexible solution to this problem has emerged with the development of step-scan interferometers. ${ }^{13-17}$ Recently, we described the implementation of step-scan FT-Raman spectroscopy with picosecond laser pulses and reported results demonstrating the first application of picosecond time-resolved FT-Raman spectroscopy. ${ }^{18}$ The time resolution of our system is $100 \mathrm{ps}$, determined by the laser pulse width, although resolution of a few picoseconds is achievable in principle by time-resolved FT-Raman spectroscopy. Preliminary Raman spectra were reported of the excited state of the highly fluorescent molecule 9,10diphenylanthracene (DPA). ${ }^{18}$

In the present paper we report the application of picosecond time-resolved FT-Raman spectroscopy to the evolution of the excited-state Raman spectrum of DPA in two solvents, cyclohexane and ethanol. This work demonstrates the potential of time-resolved FT Raman spectroscopy for probing excited-state photochemistry, photophysics, and vibrational dynamics.

\section{EXPERIMENTAL METHODOLOGY}

Picosecond time-resolved FT-Raman spectra of the first excited singlet state of DPA were measured with the apparatus shown schematically in Fig. 1. Laser pulses at 355 $\mathrm{nm}$ were focused into the sample to excite DPA into its first excited singlet state, and probe pulses at $1064 \mathrm{~nm}$ were used to generate Raman scattering. Laser pulses of 100 -ps duration at $1064 \mathrm{~nm}$ were produced by a modelocked and Q-switched (at $2 \mathrm{kHz}$ ) Nd:YAG laser (Coherent Antares).

A single pulse from each Q-switched pulse train was selected with a Pockels cell (Medox Electro Optics Inc.). These pulses were separated from the remaining pulses in the pulse train by a Glan laser polarizer. The single pulses were directed into a lithium triborate (LBO) second-harmonic generator to produce $532-\mathrm{nm}$ radiation. The unconverted 1064-nm single pulses were separated from the 532-nm pulses with a dichroic mirror and directed into an optical delay line for use as the probe beam. The pulse trains (minus the selected single pulses) were delayed $13 \mathrm{~ns}$ (the time interval between pulses in the pulse train), and combined with the 532-nm pulses in a $\beta$-barium borate (BBO) crystal to generate $355-\mathrm{nm}$ pulses. Substantial losses in both pump and probe pulse energies were observed if the third-harmonic generator was placed directly after the second-harmonic generator. The method described above, by using a second $1064-\mathrm{nm}$ pulse from each pulse train for conversion of the $532-\mathrm{nm}$ pulses by sum frequency generation to produce the $355-\mathrm{nm}$ third 


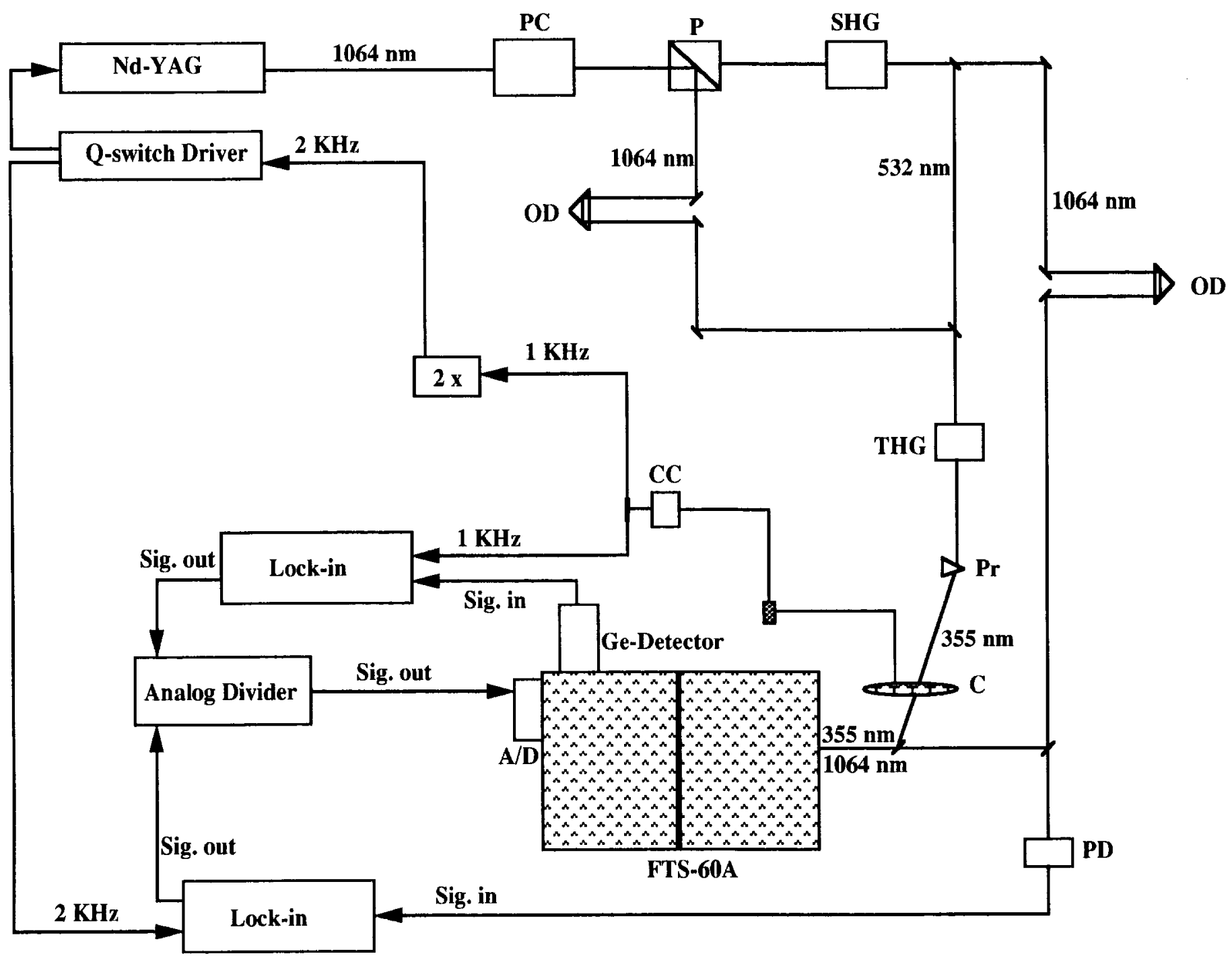

FIG. 1. Schematic diagram of the time-resolved Fourier transform Raman system. PC, Pockels cell; P, polarizer; SHG, second-harmonic generator; OD, optical delay line; THG, third-harmonic generator; Pr, prism; C, chopper; CC, chopper controller; PD, photodiode. [Reprinted from Spectrochim. Acta 50A, 1825 (1994) with kind permission from Elsevier Science Ltd., Kidlington, U.K.]

harmonic, increases both pump and probe pulse energies significantly, from $15 \mu \mathrm{J}$ to $35 \mu \mathrm{J}$ at $355 \mathrm{~nm}$, and from $100 \mu \mathrm{J}$ to $175 \mu \mathrm{J}$ at $1064 \mathrm{~nm}$. The time delay of $1064-$ $\mathrm{nm}$ probe pulses with respect to $355-\mathrm{nm}$ pump pulses was controlled by a stepping-motor-driven delay line (Unislide).

The pump and probe beam was focused into the sample inside the BioRad FTS-60A Raman module. The sample was flowed continuously from a nozzle at a given speed so that each pulse excited a fresh sample. Pump and probe beams were focused directly onto the freely flowing sample. The BioRad FTS-60A/896 interferometer was operated in step-scan mode with a step rate of $100 \mathrm{~Hz}$ during the time-resolved Raman measurement. The interferometer signal was detected with a Ge detector (North Coast Scientific).

The pump-pulse repetition rate was reduced to half that of the $2-\mathrm{kHz}$ probe pulses with a chopper. The Nd:YAG laser Q-switch and the chopper were synchronized by triggering the laser externally at twice the chopper frequency. The signal from the detector was processed by a lock-in amplifier referenced at $1 \mathrm{kHz}$ in order to selectively amplify changes induced in the signal by the pump pulses. A reference signal was generated by probe pulses detected by a photodiode and amplified by a lock-in amplifier referenced at $2 \mathrm{kHz}$. The Raman signal output was divided by the reference in order to cancel out laser drift. The output of the analog divider was directed into the FTS-60A A/D converter for processing.

Excitation and probe beam average powers were nearly constant $( \pm 2 \mathrm{~mW})$ before and after the data collection at any time delay. The spectral resolution and the total data collection time at each time delay were $8 \mathrm{~cm}^{-1}$ and 6.5 $\mathrm{h}$, respectively. A band generated by $355-\mathrm{nm}$ pump pulses and attributed to emission from the Rayleigh-line rejection filter was removed from each time-resolved spectrum by subtraction of the spectrum recorded with pump pulses alone.

Continuous wave (cw) Raman spectra, excited by radiation from a diode-pumped Nd:YAG laser (Laser Diode LDP1000-8), were recorded in the rapid-scan mode of the interferometer. 


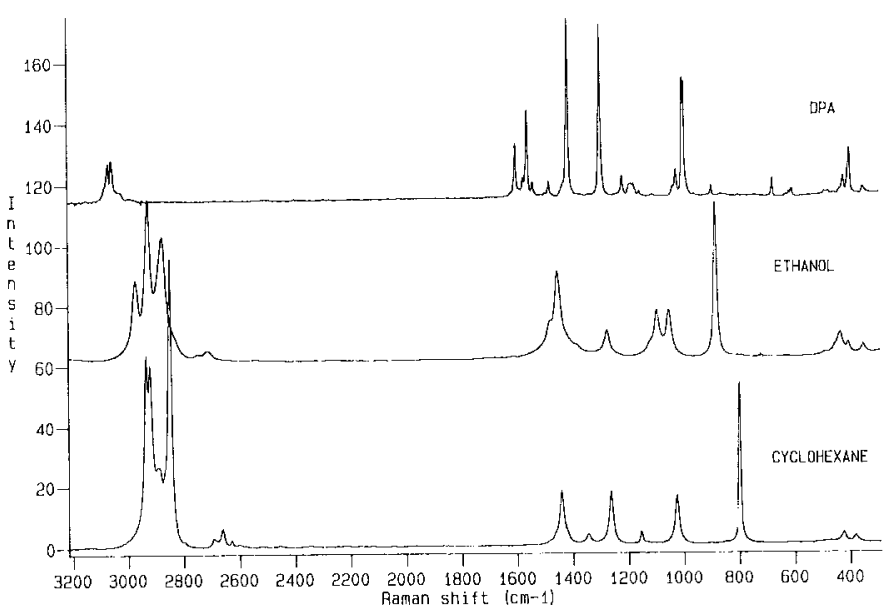

Fig. 2. Fourier transform Raman spectra of 9,10-diphenylanthracene in cyclohexane (top), and the pure solvents ethanol (center) and cyclohexane (bottom). The ground-state Raman spectrum of diphenylanthracene was excited with $450 \mathrm{~mW}$ of $\mathrm{cw}$ 1064-nm radiation (6000 scans at $2-\mathrm{cm}^{-1}$ resolution). The Raman spectra of the solvents were generated with Q-switched Nd:YAG pulses with the interferometer operated in step-scan mode (100 steps/s).

\section{RESULTS AND DISCUSSION}

The ground-state FT-Raman spectrum of DPA in cyclohexane, generated with $\mathrm{cw} 1064-\mathrm{nm}$ excitation, is shown in Fig. 2. The frequencies of the observed Raman bands are tabulated in Table I. The Raman spectra of the solvents cyclohexane and ethanol used in these studies are also shown.

DPA in Cyclohexane. Picosecond pump/probe timeresolved FT-Raman spectra of DPA in cyclohexane are shown in Fig. 3 for four different time delays between pump and probe pulse. Positive bands report an increase in Raman scattering with respect to the signal generated by probe pulses alone, and negative bands show a decrease. The spectrum at a time delay of -200 ps (i.e., the probe pulse preceded the pump pulse by $200 \mathrm{ps}$ ), contains slight increases in signal at 662,1377 , and $1585 \mathrm{~cm}^{-1}$. We attribute these bands to a weak excited-state Raman

TABLE I. Ground and excited singlet-state Raman frequencies of 9,10diphenylanthracene.

\begin{tabular}{ccc}
\hline $\begin{array}{c}\text { Ground state } \\
\left(\mathrm{cm}^{-1}\right)\end{array}$ & $\begin{array}{c}\text { Excited state in } \\
\text { cyclohexane }\left(\mathrm{cm}^{-1}\right)\end{array}$ & $\begin{array}{c}\text { Excited state in } \\
\text { ethanol }\left(\mathrm{cm}^{-1}\right)\end{array}$ \\
\hline 398 & 391 & 390 \\
607 & & \\
676 & 662 & \\
896 & 885 & \\
995 & 995 & \\
1000 & & 1174 \\
1023 & 1175 & \\
1178 & & 1297 \\
1186 & 1294 & 1375 \\
1219 & 1377 & 1419 \\
1298 & 1416 & \\
1416 & 1497 & \\
1482 & & \\
1540 & 1585 & \\
1560 & & \\
1602 & & \\
3057 & & \\
3069 & & \\
\end{tabular}

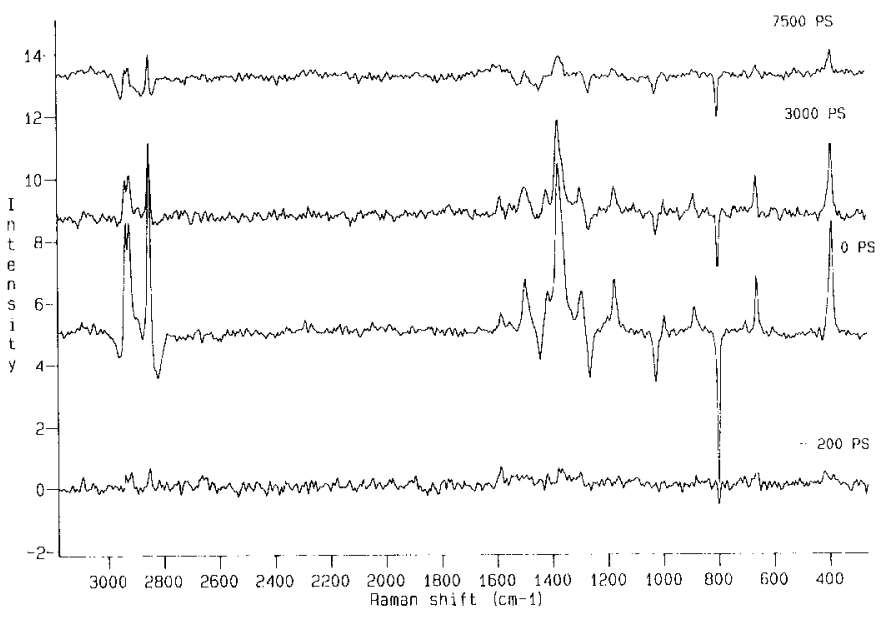

Fig. 3. Time-resolved Fourier transform Raman spectrum of 9,10diphenylanthracene in cyclohexane at time delays (from bottom) of $-200,0,3000$, and $7500 \mathrm{ps}$. The concentration of diphenylanthracene in cyclohexane was about $10 \mathrm{mM}$. The Fourier transform interferometer was stepped at $100 \mathrm{steps} / \mathrm{s}$. A total of 180 scans were summed at each time delay.

signal due to a small degree of overlap of the 100-ps wide full width at half-maximum (FWHM) pump and probe pulses. Strong positive and negative bands appear in the spectrum obtained at zero time delay $( \pm 100 \mathrm{ps})$, where the pump and probe pulses arrive simultaneously at the sample. The emergence of these bands between -200 ps and 0 ps is consistent with the 100-ps time resolution of this instrumentation. The excited-state concentration engendered by the pump pulses is estimated to be about 3 $\mathrm{mM}$.

At longer time delays ( 3000 and $7500 \mathrm{ps)}$ these signals diminish with a lifetime of 5 to $6 \mathrm{~ns}$. These bands can therefore be assigned to the excited singlet state $S_{1}$ of DPA. The decay may appear slightly shorter than the 7.6ns excited-state lifetime reported for DPA in cyclohexane ${ }^{19}$ because of uncertainties in the relative Raman intensities from one time delay to the next resulting from drifts in laser power or overlap between pump and probe pulses, or because of quenching of DPA excited states. The observed excited singlet Raman frequencies of DPA are displayed in Table I. Although $S_{1}$ DPA Raman modes appear as positive bands with strong intensity, we observe no ground-state DPA modes in the time-resolved spectra in Fig. 3. These might be expected to appear as negative bands due to decreased Raman scattering ("bleaching") from the DPA ground state. The fact that $S_{0}$ Raman modes of DPA are not observed while $S_{1}$ modes are suggests that $S_{1}$ Raman scattering of DPA is resonantly enhanced at $1064 \mathrm{~nm}$.

The negative bands at $800,1027,1265$, and $1443 \mathrm{~cm}^{-1}$ match the frequencies of the strong solvent bands in this region of the spectrum (see Fig. 2). The intensity decrease in these bands shows that solvent Raman scattering is diminished after excitation of the sample. The intensities of these bands appear to recover with the same lifetime as the decay of the excited-state Raman bands. The observed intensity decrease in these negative bands was about $4 \%$ of the total intensity of the cyclohexane Raman bands. This observation can be understood as a result of excitedstate absorption of the 1064-nm pulses following exci- 


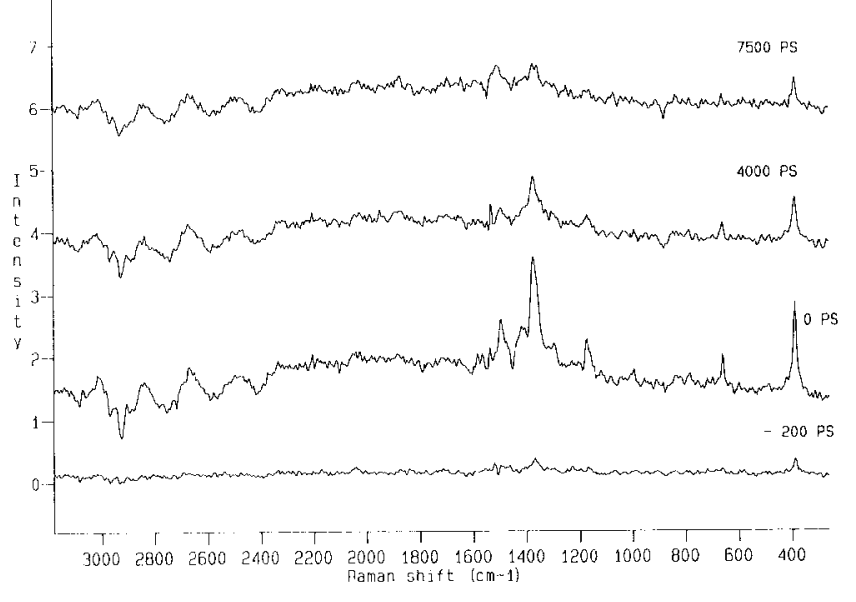

FIG. 4. Time-resolved Fourier transform Raman spectrum of 9,10diphenylanthracene in cyclohexane at time delays (from bottom) of $-200,0,4000$, and $7500 \mathrm{ps}$. The concentration of the sample was 3$5 \mathrm{mM}$. The Fourier transform interferometer was stepped at 100 steps/ s. A total of 180 scans were summed at each time delay.

tation of DPA at $355 \mathrm{~nm}$, which reduces the intensity of the probe beam in the sample. The solvent Raman signal decreases accordingly.

The transmitted intensity of the 1064-nm beam was directly measured by a photodiode, and observed to decrease by about $4 \%$ immediately following the pump pulse. In anthracene, an excited singlet ${ }^{\prime} B_{3 g}$ state $\left(S_{3}\right)$ exists at approximately 8200 to $9100 \mathrm{~cm}^{-1}$ above the ${ }^{1} B_{1 u}\left(S_{1}\right)$ state. ${ }^{20,21} \mathrm{~A}$ similarly placed state is anticipated in DPA. Consequently, the $1064-\mathrm{nm}$ probe $\left(9400 \mathrm{~cm}^{-1}\right)$ is resonant with the $S_{3} \leftarrow S_{1}$ transition. This observation confirms the suggestion that the excited-state Raman bands of DPA are observed by resonance Raman scattering.

An apparently anomalous feature in the time-resolved Raman spectra of DPA in cyclohexane appears in the positive bands at 2851,2924 , and $2936 \mathrm{~cm}^{-1}$. These frequencies are characteristic of aliphatic (not aromatic) $\mathrm{C}-\mathrm{H}$ stretching vibrations, and match exactly the groundstate Raman frequencies of cyclohexane (see Fig. 2). These positive bands indicate an increase in cyclohexane $\mathrm{C}-\mathrm{H}$ Raman scattering and appear to be superimposed on a negative component which ensues from the decrease in the intensity of the 1064-nm probe beam discussed above. The enhanced solvent scattering in this region may reflect an interaction of the solvent with the excited state of DPA. Although excitation of DPA at $355 \mathrm{~nm}$ must cause some heating of the solvent surrounding the excited molecule, we think it unlikely that thermal effects can explain the observations in this region for the following reasons: (1) solvent heating would affect all solvent bands, not just $\mathrm{C}-\mathrm{H}$ stretching modes; and (2) the enhanced solvent Raman scattering does not appear in ethanol (see below) and seems to depend on the nature of the solvent.

DPA in Ethanol. Time-resolved Raman spectra were also measured for DPA in ethanol. These spectra are shown in Fig. 4. At a negative time delay of -200 ps, a weak excited-state Raman signal can be observed as in Fig. 3, because of a small overlap between the probe and pump pulses. At zero time delay, excited singlet-state Raman bands of DPA are observed at frequencies that match closely the frequencies found for DPA in cyclo- hexane (see Table I). The excited-state concentration was estimated to be $0.5 \mathrm{mM}-1.0 \mathrm{mM}$. The time-resolved Raman signals are weaker for these spectra than in Fig. 3 because of a lower excited-state concentration of DPA. The excited-state Raman bands decay with roughly the same lifetime as in cyclohexane. Negative bands again appear, corresponding to ground-state Raman frequencies of ethanol.

In contrast to the spectra of DPA in cyclohexane, the ethanol solvent bands around $2928 \mathrm{~cm}^{-1}$ remain negative. Apparently the solute-solvent interaction responsible for increased solvent intensity for DPA in cyclohexane is not active in ethanol. A larger background is observed relative to the Raman signal than in Fig. 3. The ripples observed in the $2300-2900 \mathrm{~cm}^{-1}$ region in Fig. 4 are attributed to interference of the background in a visible blocking filter. The intensity of this interference pattern was found to increase or decrease with the sample concentration, suggesting that the background derives from DPA fluorescence, which may be absorbed by an optical filter which subsequently re-emits in the near infrared region.

Ground and Excited-State Raman Modes of DPA. The observed ground and excited singlet state Raman frequencies of DPA are tabulated in Table I. A ground-state UV resonance Raman spectrum $\left(11-\mathrm{cm}^{-1}\right.$ resolution) of DPA excited at $254 \mathrm{~nm}$ has been reported previously. ${ }^{22}$ Several of the major ground-state bands in Fig. 2 (398, $676,896,1178,1298$, and $1560 \mathrm{~cm}^{-1}$ ) were also observed in the UV resonance Raman spectrum.

The excited-state vibrational frequencies of DPA have not been previously reported. Most of the observed excited-state frequencies appear not to be greatly changed from ground-state frequencies, suggesting only minor structural changes between $S_{0}$ and $S_{1}$. The strong $S_{1}$ Raman bands observed in Figs. 3 and 4 are most likely totally symmetric modes, which are expected to be most strongly enhanced in resonance Raman spectroscopy. Although a normal-mode analysis of DPA is not available, a normal-mode calculation has been reported for the anthracene ground state. ${ }^{23}$ Several of the strongest $S_{1}$ Raman bands $\left(391,1175,1377\right.$, and $\left.1497 \mathrm{~cm}^{-1}\right)$ seem to correlate with totally symmetric anthracene modes involving symmetric distortion of the $\mathrm{C}-\mathrm{C}$ framework in the anthracene plane. The band at $662 \mathrm{~cm}^{-1}$, on the other hand, is associated with substitution at the 9 position. ${ }^{22}$ Bands at $995-1000 \mathrm{~cm}^{-1}$ in the ground and excited states are likely associated with the phenyl rings.

\section{CONCLUSION}

We have used step-scan Fourier transform Raman spectroscopy with picosecond time resolution to probe excited-state vibrations and interactions with solvent of DPA. DPA, with a fluorescence quantum yield of nearly unity, would present a considerable challenge to timeresolved resonance Raman spectroscopy with visible probe pulses. Raman excitation in the near-infrared disengages the Raman scattering from fluorescence in the visible spectral region. Further application of this technique can be anticipated in studies of excited-state conformations, excited-state reactions, and short-lived ground-state reaction intermediates. 
At present, we have applied time-resolved FT-Raman spectroscopy only to molecules with an excited-state transition resonant with the probe wavelength. It is not clear how restrictive a limitation this condition represents, since not many measurements of excited-state absorption in the near-IR are available. Many molecules with lowestlying excited states in the visible or near-UV (e.g., polycyclic aromatic compounds with two or more rings) are likely to have excited-state transitions in the near-IR, and therefore to exhibit resonance enhancement of excitedstate Raman scattering in the near-IR. How generally applicable the method of time-resolved FT Raman with near-IR excitation might be is still to be determined.

While the signal-to-noise $(\mathrm{S} / \mathrm{N})$ obtained here is comparable to that achieved in many visible time-resolved Raman measurements, the data collection time $(\sim 6 \mathrm{~h})$ is longer than that realized by visible picosecond time-resolved Raman methods in favorable circumstances. ${ }^{3-5}$ This difference is a result of the Raman scattering efficiency in the near-IR and the performance of the germanium detector, and is not a consequence of step-scan operation, which causes little or no degradation of S/N. ${ }^{18}$ Improved $\mathrm{S} / \mathrm{N}$ performance may also be achievable by selection of filters that block UV and visible fluorescence and do not re-emit. Improved time resolution can be achieved by compression of the near-IR pulses or by use of lasers (e.g., $\mathrm{Ti}$ : sapphire) with intrinsically shorter pulse widths.

\section{ACKNOWLEDGMENTS}

This work was supported in part by NSF under EPSCoR grant No. 9255223 and by the University of Kansas General Research allocation \#3614. This work also received matching support from the State of Kansas EPSCoR program. The FT-Raman instrumentation was funded by Grant CHE-9023773 from the NSF Chemical Instrumentation Program.

1. Time-Resolved Laser Raman Spectroscopy, D. Phillips and G. H. Atkinson, Eds. (Harwood, Chur, Switzerland, 1987).
2. Time-Resolved Vibrational Spectroscopy V, H. Takahashi, Ed. (Springer, Berlin, 1991); Time-Resolved Vibrational Spectroscopy VI, A. Lau, F. Siebert, and W. Werncke, Eds. (Springer, Berlin, 1993).

3. K. Iwata, S. Yamaguchi, and H. Hamaguchi, Rev. Sci. Instrum. 64, 2140 (1993); K. Iwata and H. Hamaguchi, Chem. Phys. Lett. 196, 462 (1992).

4. W. L. Weaver, L. A. Huston, K. Iwata, and T. L. Gustafson, J. Phys. Chem. 96, 8956 (1992); R. M. Butler, M. A. Lynn, and T. L. Gustafson, J. Phys. Chem. 97, 2609 (1993).

5. J. Qian, S. L. Schultz, G. R. Bradburn, and J. M. Jean, J. Phys. Chem. 97, 10638 (1993).

6. P. A. Anfinrud, C. K. Johnson, R. Sension, and R. M. Hochstrasser, "Ultrasfast Spectroscopic Methods," in Applied Laser Spectroscopy: Techniques, Instrumentation, and Applications, D. L. Andrews, Ed. (VCH, New York, 1992), Chap. 10, p. 410.

7. T. L. Gustafson, D. M. Roberts, and D. A. Chernoff, J. Chem. Phys. 79, 1559 (1983).

8. T. Hirschfeld and B. Chase, Appl. Spectrosc. 40, 133 (1986).

9. V. M. Hallmark, C. G. Zimba, J. D. Swalen, and J. F. Rabolt, Spectroscopy 2, 39 (1990).

10. S. Nie, K. L. Bergbauer, J. J. Ho, J. F. R. Kuck, Jr., and N. T. Yu, Spectroscopy 5, 24 (1990).

11. P. J. Hendra, C. Jones, and G. Warnes, Fourier Transform Raman Spectroscopy: Instrumentation and Chemical Applications (Ellis Harwood, New York, 1991).

12. C. K. Johnson and R. Rubinovitz, Appl. Spectrosc. 44, 1103 (1990).

13. R. A. Palmer, Spectroscopy 8, 26 (1993).

14. R. A. Palmer, J. L. Chao, R. M. Dittmar, V. G. Gregoriou, and S. E. Plunkett, Appl. Spectrosc. 47, 1297 (1993).

15. I. Noda, A. E. Dowrey, and C. Marcott, Appl. Spectrosc. 47, 1317 (1993).

16. W. Uhmann, A. Becker, C. Taran, and F. Siebert, Appl. Spectrosc. 45, 390 (1991).

17. J. J. Sloan and E. J. Kruus, "Time-Resolved Fourier Transform Spectroscopy," in Time Resolved Spectroscopy, Advances in Spectroscopy, Vol. 18, R. J. H. Clark and R. E. Hester, Eds. (Wiley, Chichester, England, 1989), pp. 219-253.

18. G. S. Jas, C. Z. Wan, and C. K. Johnson, Spectrochim. Act. 50A, 1825 (1994).

19. A. Maciejewski and R. P. Steer, J. Photochem. 35, 59 (1986).

20. B. Dick and G. Hohlneicher, Chem. Phys. Lett. 83, 615 (1981).

21. P. R. Salvi and G. Marconi, J. Chem. Phys. 84, 2542 (1986).

22. S. A. Asher, Anal. Chem. 56, 720 (1984).

23. K. Ohno, J. Mol. Spectrosc. 72, 238 (1978); ibid., 77, 329 (1979). 